\title{
New Microscopy Techniques Facilitate the Deorphanization of GPCRs
}

\author{
A. F. Hoffman
}

Roche Discovery Technologies, Hoffmann-La Roche Inc., 340 Kingsland Street, Nutley, NJ 07110

During the past five years the field of microscopy has expanded to embrace numerous new technologies that have emerged to facilitate the early drug discovery objectives of defining new therapeutic targets and identifying potential new chemical entities. The challenges of utilizing microscopy in the early drug discovery process has been addressed with state-of-the art screening instrumentation, large-scale expression systems, high speed image analysis processing and the efficient databases that manage, archive and enable data mining. This presentation will highlight the approach that these High Content Screening tools have made possible within a systems-based project designed to deorphanize G protein coupled receptors (GPCRs).

We sought to identify orphan GPCRs by querying for transmembrane consensus domains clustered around previously targeted receptors known to modulate energy balance using a bioinformatics approach. Candidate genes with human and murine homologues were defined. An expression profiling analysis of each gene was conducted in normal and diseased human tissues to select those primarily expressed in brain. After identification of selected gene sequences the modulation of gene expression from specific brain areas of normal and diet inducted obese mice was performed. Differential expression quantified by PCR was accomplished using laser capture microscopy in which discrete brain regions were isolated, removed and analyzed. The most promising candidate proteins were expressed using the Norak Transfluor Technology. This technology employs the use of a GFP-tagged beta-arrestin which complexes with the receptor and upon activation results in the redistribution of the receptor:GFP-arrestin complex. Characteristics of each orphan cell line were varied in regard to the degree of basal constitutive activity, and the degree of stimulated activity in response to a baculovirus transduced $\mathrm{G}$ protein regulated kinase.

Four distinct high throughput imaging technology platforms were employed to quantify the fluorescent redistribution cellular phenotypes as we screened our main compound library for agonists and inverse agonists. Descriptions of these automated platforms are detailed. This includes the acquisition modes, image analysis algorithms and data processing. Data visualization tools such as IDBS's Spotfire and Evotec's A+ software have been used to define the resulting "active compounds". Operational metrics and screening comparisons are shown including multi-parameter data. A description of the data management system provides the methods used for storing, archiving, and retrieval of both image and data files for reanalysis and bioinformatics queries.

In conclusion, new automation-assisted cell-based high content platforms have provided an optimal combination of throughput and context for functional screening of candidate drug targets as illustrated by the orphan GPCR project described. We were able to identify novel compounds of varying specificity and activity for the oGPCRs and compounds which activated unexpected barrestin translocation phenotypes. These image-based technologies, instrumentation, cell lines and reagents, afford researchers increased flexibility in studying cellular systems using systems biology approaches. 

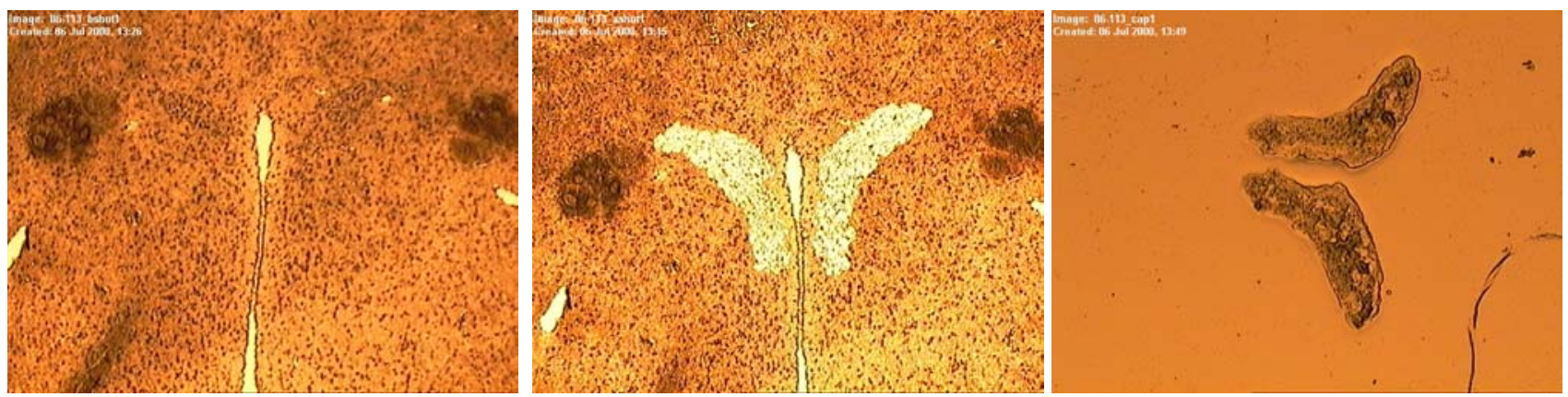

Figure 1. Laser capture microscopy dissection of the paraventricular nucleus from mouse brain. Sections were used to quantify total RNA yields correlating to the orphan GPCRs after primer amplification.

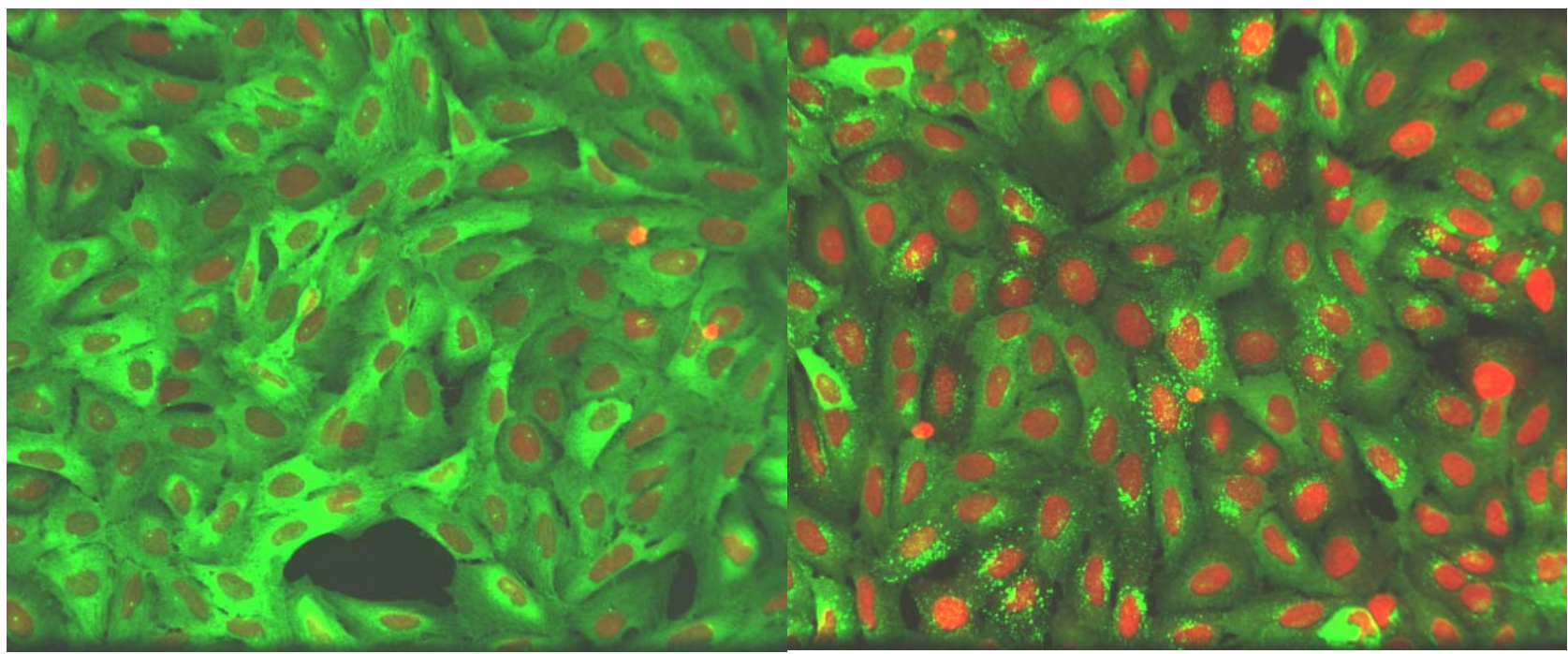

Figure 2. Comparison of Norak Transfluor GFP beta-arrestin containing U 2-OS cells stably transfected with oGPCR \#3 under basal conditions (left) and after receptor internalization using baculovirus transduced $\mathrm{G}$ protein regulated kinase treatment (right). Draq5 nuclear labeling, magnification 20x, image acquisition on the Evotec Opera Automated Confocal Platform.

Figure 3. Quantification of receptor internalization as measured by \% Phase 3 responding cells of 10 stably transfected oGPCR clones and one clone of B2-adrenergic receptor. Image acquisition and analysis was performed using the Cellomics ArrayScan 3.1 High Content Imaging Platform and its associated GPCR Bioapplication.

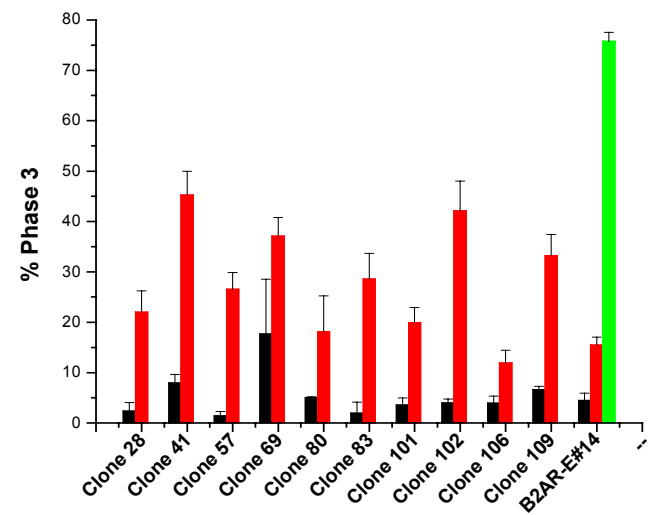

